\title{
New Resin Cements Agents in Conservative Dentistry: Indications and Possible Risks. Rev Literature
}

\author{
Moradas Estrada $\mathbf{M}^{*}$
}

Associate Professor, Conservative Dentistry Service of the University Clinic of the University of Oviedo, Spain

${ }^{*}$ Corresponding author: Moradas Estrada M, Associate Professor, Conservative Dentistry Service of the University Clinic of the University of Oviedo, Spain, Tel: 646552313, E-mail: marcosmords@gmail.com

Citation: Moradas Estrada M (2017) New Resin Cements Agents in Conservative Dentistry: Indications and Possible Risks.Rev Literature. J Mater Sci Nanotechnol 6(2): 201

Received Date: May 30, 2017 Accepted Date: January 08, 2018 Published Date: January 11, 2018

\begin{abstract}
The demand for aesthetic dentistry is increasing in today's society, aspect and images are increasingly important. Among the wide variety of restorative techniques exist entities, direct resin-based composite techniques are one of the best treatment options and have gained wide acceptance. Composite cements are one of the most used materials in any specialty of dentistry, more so when we are interested in being able to join/adhere two or more structures of heterogeneous nature. Over the last twenty-five years, multiple and distinct types of composite cement have been described, with no indication protocol for different clinical situations. Therefore, we have proposed to answer throughout this review which cement would be the most indicated in the clinical situations that occur most in clinical on the basis of its chemical-mechanical properties.
\end{abstract}

Keywords: Luting agent; Resin cement; Adhesive cement; Onlays; Inlays; Composite

\section{Introduction}

The use of indirect restorations anchored with adhesive procedures is a fundamental part of current dental restorative treatments. Crowns with or without metal, inlays, onlays, veneers, posts, are routine treatments in the daily clinic and are summarized to the capacity of an artificial material, of different nature, being able to adhere to the tooth, with functional, aesthetic, Non-iatrogenic and long-term [1]. Part of these qualities will be given by the cementitious interface, and especially the resin composite interface $[2,3]$. Composite cements are mixtures of polymerizable monomers of methacrylate, dimethacrylate and polymethacrylate in an organic matrix (Bis-GMA, TEGMA, UDMA) [4]. For self-adhesive resin cements, highly acid monomers, multifunctional monomethacrylates and methacrylates are added to the blend. Other additives may also be included to increase the strength of adhesion to metals, such as 4-MET, whereby the composition is very similar to that of a normal composite $[4,5]$. Some manufacturers have included fluorinated cement components, although this practice is of dubious value due to hydrophobicity of resins, since the mechanisms of fluoride releasing and maintaining a low water absorption are mutually exclusive [5]. In spite of their great adhesion power, these cements are very sensitive to the technique, saying that $50 \%$ of the success is in the hands of the operator (cements manipulation and previous maneuvers to perform on the preparations, both the tooth and restoration), being more sensitive cements total etch the self-recorded [6,7]. The flexural strength they support is greater than that of other cements such as glass ionomer cement, zinc phosphate or resin modified glass ionomer cement. Likewise, the modulus of elasticity is smaller than that of zinc phosphates, but higher than glass ionomer cement and the resin-reinforced glass ionomer itself. The radiopacity is similar to that of enamel, much lower than that provided by zinc phosphate [8]. In addition, microfiltration was shown to be lower than that of resin modified glass ionomers [9]. In contrast, debris is more difficult to remove than conventional cements, and those that are not self-adhesive require a large number of steps for proper cementation [10].

In the early 1980s, products described as hybrid glass ionomer cements and composite resin were introduced as "resin-modified glass ionomer", this material has very good mechanical properties compared to zinc phosphate and some Conventional glass ionomer cements [11]. The resin modified glass ionomer cementation agents are a chemical melting of the glass ionomer and the resin by an acid-base reaction between the aluminum silicate glass powder and an aqueous solution of polyalkenoic acids modified with methacrylate groups as the initiator Chemical free radical polymerization of the methacrylate units. Resin-modified glass ionomer cements behave as an intermediate between resins and glass ionomer cements.

Classification of resinous cements Resin cements can be classified according to several criteria, among which are: a. By the size of its particles, b. By its form of activation, c. For its adhesiveness to by the size of its particles [12,13]: 


\section{Materials and Methodology}

A descriptive bibliographic review of the evidence provided in indexed articles and other bibliographic sources, such as books, theses and others, has been made. It was performed using keywords, dual curing resin, operative dentistry, acondicioner agent, lutting agent, etching, cement, glass ionomer, search the online source is MED LINE, obtaining a total of 355 results. These were analyzed and after verifying whether or not they met the criteria for inclu sion/exclusion, which were included publication year from 2003 to 2012, include a historical article for their excellent contributions; Bibliographical review article, meta-analysis or systematic review (thus discarding cases/controls or similar); Journal indexed between the second and third percentile; Works not financed by commercial houses to avoid the introduction of bias; The articles with the presence of methodological errors type I and II are also eliminated. Finally were 55 articles used to make the present review.

\section{By the size of its particles}

Microparticulate: Its inorganic filler particles have an average size of $0.04 \mathrm{um}$ and its percentage of approximately $50 \%$ by volume $[12,13]$.

Micro hybrids: They constitute the majority of the resinous cements that are in the dental market. The average size of their inorganic filler particles is about $0.04 \mathrm{um}$ to $15 \mathrm{um}$, which is incorporated in a percentage of about 60 to $80 \mathrm{volume} \%$ [12,13]. According to literature data, the best results are obtained with the cements that have microhybrid particles in their composition, because their polymerization shrinkage is lower and they have a medium viscosity, which allows an adequate settlement of the restoration $[12,13]$.

\section{By their form of activation:}

Resin cements can be activated chemically, photoactivated and even present double activation; Ie dual activation [12,13].

Chemically activated resinous cements Chemical activation, despite not allowing adequate working time, promotes a polymerization characterized by a high degree of conversion of monomers into polymers, so it is considered the best option, within resin cements, For cementing non-metallic adhesive posts and restorations or metal prosthetic pieces [12]. After mixing the base paste with its catalyst, a peroxide-amine reaction is initiated which initiates the curing reaction. These materials usually do not present aesthetic characteristics, since most of the times they have a opaque white appearance and few color options [12,13]. Composite resin cements are chemically bonded to composite restorative materials and to silanized porcelain. Adhesive resins increase the breaking strength of ceramic materials that can be conditioned and silanized.

\section{Cemented Adhesive}

The cementation of indirect restorations in fixed prostheses (PF) is one of the most important steps in achieving adequate retention, resistance and sealing of the interface between the restorative material and the tooth [14]. The Long-term restoration in the mouth.

With adhesive cementation all the described objectives are achieved more effectively than with other types of cement. In fact it is the only type of cementation that Can be used for restorations with low friction retention because of their high bond strength $[15,16]$. 1-4 The diversity of restorative materials used today requires specific cements and systems to achieve effective and durable adhesive bonding. Ceramic materials increasingly take a leading role in daily dentistry and it is necessary to employ a specific cementing system [17-19].

The different adhesive systems must be understood in order to select the most suitable for each ceramic material and each type of restoration [20].

\section{Classification of adhesive cements 5}

If it is a question of organizing the cements according to their capacity of adhesion would be considered two great blocks:

Ionomer Glass Cements: Adhesive cements are considered as some degree of chemical bonding is produced by covalent bonds.

- Ionomer Glass Cements (CIV).

- Ionomer Glass Cements Modified With Resin.

Resin Cements: They are those that provide an adhesion by mechanism of micromechanical retention in enamel and by hybridization in dentin. It is the strongest and most effective dentin adhesion system. In the present work we are going to consider them as adhesive cement itself.

- Adhesive Resin Cements Without Filling.

- Cementos D And Composite.

- Autogrants.

- No To Receivers.

- Components (Resin Modified With CIV).

- Cementos D 


\section{Variety and Composite Cement Types Existing in the Market}

Composite cements can be classified according to three variants [21]:

- Type of polymerization

- Type of dental surface treatment

- Filling material

\section{Classification according to the type of polymerization}

Cements of Chemical Curing Composite: They have a peroxide-amine reaction, which starts with the mixture of the base paste and the catalyst paste, which limits the working time for the professional, that is, to settle the restoration and eliminate excess cement. They are not usually aesthetic because of their opacity [22]. They are useful when restorations do not allow light-curing light, such as metal crowns or endodontic posts [23]. Also these cements have 25\% less hardness than dual cure [3,24-28].

Cements of Photopolymerizable Composite: Its action is the presence of photoinitiators allows as camphorquinone, activated in the presence of light with a wavelength around 470 nanometers and triggers the polymerization of the material. They allow a much longer working time than the other types of cement, which facilitates its use [29]. In addition, the color stability is higher compared to chemical or dual curing cements. Consideration must be given to the need for sufficient light transmission through the restoration to polymerize the cement. For example a porcelain veneer absorbs $40-50 \%$ of light emitted on it, and if it measures more than $0.7 \mathrm{~mm}$ will not achieve a polymerization of $100 \%$ [30].

Dual Cure Composite Cement: These begin to polymerize when the base paste and the catalyst paste are brought into contact [29]. In addition, by light, the photoinitiators present in the cement are activated, because in case of not using light the maximum hardness value of the cement will not be reached until the seventh day, with the risks that this entails for the physical and chemical stability of the restoration [13]. It is important to consider the thickness of the restoration adherend, because although does not affect the chemical polymerization it does for the photopolymerization. From $4 \mathrm{~mm}$ thick cement has been observed as the restoration hardness decreases up to 50\%, even measured one week after curing 813). Some of these cements present a try-in system, which is a soluble paste that can be used as a test before final cementation [29]. These cements are very useful in metal restorations, where light can be used to seal the margins quickly [31]. Studies such as Nuray et al. [8] show that it is the best cement due to the combination of mechanical, With a high $\mathrm{pH}$, which again highlights the need for photopolymerization to achieve $100 \%$ of the properties that give us this type of cement.

\section{According to the treatment of the dental surface}

Total Engraving Cements: They are cements that require full preparation of the surface of the tooth prior to cementing agent. First an engraving of orthophosphoric acid 30 to no more than $40 \%$ is used to record dentin and enamel, many authors point to the classic dose of composite fillings or sci qu and the 33-35\%. Thereafter, an adhesive agent is applied to bond cement and dental substrate [32]. In this way, by being able to modify the adhesive surfaces specifically and heterogeneously on both sides, we achieve the highest adhesion force possible, which requires the greatest number of steps, better and better control of the technique and a high cost [11].

Self-Adhesive Grinding Cements: A simple way to save steps and therefore time and cost, is the use of cements that save a step from the application of etching acid to the primer, although not free from disadvantages as we will mention later.

Self-Adhesive Cements-Self-Adhesive: These types of cements combine the composition of glass ionomer cements and conventional composite cements [33]. They can be bonded to dentin, enamel or ceramic without the need for a separate adhesive. They have the ability to adhere to the tooth without the need for engraving, since they contain in their composition orthophosphoric acid. Once the mixing is started, the phosphoric acid reacts with the filler material in the presence of water to dentin, forming adhesive [11]. Although the use of a primer, together with a refined restoration surface conditioning technique, would not be required for adhesion, the adhesion force between the dentin and the restorative material is considerably increased [12].

\section{Depending on the filling material}

Cements of Composite Without Filling: It is a self-etching adhesive resin with 4 META monomer (metal bonding monomer), without inorganic filler which reduces the mechanical properties of the cement [34,35]. It is a cement that requires acid etching but which binds to the porcelain without the necessity of using hydrofluoric acid since the silane has a special composition. For comprises phosphate monomer particularly suitable for bonding of metal materials, but can also bind porcelains and resins [36,37].

Cements of Composite with a Little Filling: It is the cements that present a greater fluidity of all, increasing thus the humectante surface and for that reason the capacity of cementing potential of the surfaces to be united. On the other hand, it has less adhesion capacity than cements with higher percentage of filler, it is more difficult to remove the remains of cements and suffer a greater number of marginal microfiltrations proportional to the decrease of the filling [38-41]. 
Cementos of Composite with a Lot of Filling: The increase in the amount of filler generates a better resistance to abrasion in the marginal zone, reducing the shrinkage of polymerization and facilitating the elimination of excess cement [31]. As for their handling, these are less fluids and special handling techniques such as the use of ultrasound [41].

\section{Clinical Applications}

Composite cements can be used for multiple performances, adhesion of posts, brackets, veneers and a long etc. All of them have one thing in common that require proper preparation of the surfaces to be treated, especially when it is a composite cement total etch. It is true that authors such as Zhang et al. [41]. show how the adhesion strength in self-etching and self-adhesive resin cements is substantially improved if there is a correct and thorough surface preparation.

\section{Adhesion to Ceramics}

The treatment of the ceramic surface depends on its composition and strength [1]. Treatments include engraving, microetching or the Rocatec7Cojet technique, among many others and diverse. The etching is only effective for ceramic containing high concentrations of silica, such as feldspathic porcelain. Special care must be taken with the use of engraving times, as it is possible to overload and reduce the adhesion of the cement to the ceramic. The engraving time goes from 20 seconds to two minutes, depending on the pottery. For the lithium disilicate, a recorded 20 seconds joined hydrofluoric acid is $5 \%$ the recommended by the manufacturer (Ivoclar) step. The microetching is recommended for high strength ceramics such as Procera All Ceram (Nobel Biocare, Yorba Linda) Cercon (Dentsply) or LAVA (3M ESPE). In addition, a 5 second microetching is recommended on the surface of the ceramic with particles of alumina less than $5 \mathrm{um}$. Not forgetting that this process is contraindicated for ceramics of low strength, as it could weaken the restoration. The Rocatec/Cojet technique is recommended for feldspathic and zirconia porcelains [10]. Thus, for cementation of veneers, it should be noted that the photopolymerizable resin cement is the material of choice for bonding the substrate of the tooth [7]. In addition, the use of resinous cementitious agents enhances the qualities of the restoration, allowing to be colored, which drastically improves qualities such as the final color of the restoration [10].

\section{Adhesion to Metal}

We need to get matrix or surface with retentions where we can join something. So to roughen the surface and increase its retention potential micretching is used with oxide particles of $50 \mathrm{uc}$, with this we achieve a micromechanical retention. The next objective will be the achievement of chemical retention, ie adhesion. We will use first as 4 META or other best known as the silane. New techniques improve and extend these two traditional routes of achieving retention, such as the tribioquímico treatment consisting in coating the surface with silica, for which the restoration surface with silica particles modified with silane is abraded.

\section{Adhesion to Fiberglass Spikes}

One of the main failures of fiberglass spikes is their de-cementation, so it is essential to use a suitable cement and adhesion technique. Composite cements are the ones of choice, as they offer superior adhesion strength to glass ionomer cements and resinmodified glass ionomer cements [42]. For correct adhesion there are three types of techniques or forms of preparation of the spike: chemical preparation with silane and chemical agents, mechanical preparation with acid etching and chemical-mechanical, when put together the two previous ones. Some manufacturers include in a single solution the silane and the adhesive, since the use of both is more effective than the use of silane alone. The cement used in this procedure is the light-curing type, since the light crosses the spike obtaining from apical to coronal a better adhesion almost $100 \%[43,44]$.

\section{Access to Barackets}

Adhesion of brackets with resin cements is described as intermediate-low being intermediate - high adhesion strength cement glass ionomer still an adhesion strength too large may lead to delamination enamel when debond the brackets [45].

\section{Adhesion of Composite Inlays-Onlays}

The union of two composites where one of the two is not new, is based on the chemical union of the free radicals of the old composite with the new one. In order to improve this bond there are different options that can be used simultaneously, such as the use of silane, the use of micro etcher etching or surface cleaning with hydrofluoric or phosphoric acid [46]. The cement of choice in this type of scale is the dual cure cement. We must not forget that the thickness of the incrustations plays a crucial role, because the light must reach the total of the cementing agent so that it reaches its maximum hardness. From 3 mm thick, hardness is drastically affected cement [47-51].

\section{Adhesion to Enamel}

For optimum adhesion, the enamel, which as is known has the potential best adhesion qualities for its highly inorganic composition, it must be specifically and using a refined technique, such as the classic: application of orthophosphoric acid 30-40\%, For 15 seconds, whereby we obtain a roughness and then condition it with adhesive agent.

\section{Adhesion to dentin}

Due to the increase in water concentration to the detriment of the inorganic phase, its potential adhesion will always be weaker 
and brittle than enamel. The engraving time is less than that of the enamel, about 15 seconds and the application of the adhesive and the type of this similar. Of course, the technique is even more sensitive.

\section{Discussion}

The total etching composite cements, that is, the conventional ones, are indicated for the cementation of: metal - ceramic crowns, metallic crowns, fixed partial dentures with or without retention, ceramic metal crown with porcelain margin, ceramic inlays, veneers Ceramics, Maryland bridges, metal spikes and fiberglass spikes [10]. It does not present any contraindication, taking into account the type of curing, ie a light-curing cement if it would be contraindicated for a metal crown.

Meanwhile cements self-etching adhesive, they would be indicated before: crowns metal-ceramic, metal crowns, fixed partial denture with high retention, crown metal ceramic margin porcelain, ceramic inlays, metal pins and dowels fiberglass. They would be contraindicated and would pose a risk to restoration and dental substrate in cases of: fixed partial crowns or prostheses with poor retention, veneers and bridges Maryland [10].

During the development of an inlay, future margins that receive direct occlusal loads of opposing teeth should be avoided, which accelerates the wear of the cementing agent that leaves marginal zones susceptible to fractures, independent of the material used [1]. Ereifej, Silikas and Watts 15 in 2009 emphasized the importance of the composite resin in this treatment because of its continuity with the cementing agent, similar composition and its ductile nature that allowed it to absorb occlusal loads better than the ceramic in these marginal zones.

Multiple studies have highlighted the importance of adequate case planning to avoid future margins in occlusive contact areas that favor marginal deterioration leading to failure of restoration.16-20 Belli, Pelka, Petschelt and Lohbauer21 in 2009 stated that Not only are the margins close to occlusal contacts affected, but food during mastication and dental abrasion can influence the marginal seal of resin cement that is free of direct occlusive forces [52-55].

In the first clinical case, in the dental organ 46 the future margins of the restoration were located in zones free of direct contact or its surroundings, possibly contributing to obtain efficient results without loss of substance in the enamel-resin interface during The time of observation [2]. In cavities where the interproximal terminal line is difficult to access as in class II, it is preferable to keep as much as possible a terminal line in enamel and not in dentin by early adhesion.

Soares22 in 2005 concluded that the composite resin in indirect and direct method had a similar behavior with marginal line in enamel, and with terminal line in dentin the indirect resin exhibited more leaks than direct. On the other hand Ferreira23 in 2008 reported a similar performance between direct and indirect restorations with composite resin with cervical margin located in enamel. In relation to the above, in the second case, the return of the mesial contact area of the right upper first molar was performed with an indirect restoration with margins located in enamel and dentin, without clinical or radiographic records of adhesive failures.

In a tooth with endodontic treatment, its fragility and high structural loss are of particular interest. A cusp coating may favor biomechanical resistance but may not necessarily be developed through restorations whose margins located below the gingiva favor soft tissue inflammation. Plotin et al 24 in 2008 in a study of 45 extracted and endodontically treated lower molars concluded that there was no significant difference in fracture resistance between direct and indirect resin.

For 2010, Jiang et al. 25 compared three-dimensional models of a lower first molar of vital teeth with endodontic treatment treated with inlay and onlay inlay designs in gold, porcelain and resin, and concluded that the higher internal tension values were observed in Non-vital teeth. In addition, the composite resin type onlay showed the best perform ance in the reduction of internal tensions $[7,28,32,33,34]$.

Similar studies also reported a significant decrease in stress in root canals with restored endodontics with inlays in composite resin compared to amalgam and gold scale. These data were in agreement with the observed performance in the type of Inlay selected for molar 16 of clinical case number two, where the onlay restoration reported optimal results free of filtration and dental fracture [25].

Based on the information obtained it was concluded that indirectly composite resins offer a reliable and predictable alternative in the clinical environment, provided that the knowledge of the advantages and limitations of the different materials involved is available [19]. It should also be considered a reasonable alternative for widely affected teeth, which in some cases are valued for different materials.

\section{Conclusion}

After analysis of the work of the literature, it can be said that within the range of composite cements, which has the highest adhesion strength is dual curing, and to reach the maximum adhesion is necessary Photopolymerization. At the same time, we can say that although self-adhesive and self-adhesive cements do not necessarily require a preparation of the surfaces to be bonded, it is evidenced as a correct conditioning, we achieve maximum adhesion, which we do not apply directly composite cement.

Another idea is clear from this review, and as the clinical act of cementation of a restoration is not to be something banal and sometimes left to the support staff, but a technical and specific right for each type of restoration And the choice of the ideal cement, is essential for the clinical survival of the restoration and dental substrate, as well as to restore function and esthetics to the patient. 


\section{References}

1. Janet L, Harrison D, Waldemar G, Simon JF (2007) Resin cements: a closer look at newly introduced cements. J Dent 3: 135-39.

2. Irfab A (2012) Cementing resturaciones aesthetic. Dent Tribune 9: 7-12.

3. El-Bradwy WA, OM-Mowafy (1995) Chemical versus dual curing of resin inlay cements. J prosthet Dent 73: 515-24.

4. Omar M, Moway Rubo MH (2000) Influence of composite inlay / onlay. Thickness on hardening of dual re cured without cements. J Can Dent Assoc 66: 147-55.

5. Pegoraro TA, Da Silva NR, RM Carvalho (2007) Cements for use in esthetic dentistry. Dent Clin N Am 51: 453-71.

6. Bruniera Anchietta R, Passos Rocha E, Oliveira de Almeida E, Freitas AC, Martinia A (2011) Bonding all ceramic restorations with two resins cement techeniques. A clinical report of three-year follow up. Eur J Dent 5: 478-85.

7. Blatz MB, Sadan A, Kern M (2003) Resin ceramic bonding: a review of the literature. J prosthet Dent 89: 268-74.

8. Taira Y, Sakai M, Soeno K, Sawase T (2013) Comparison of four primers and an isocyanate silane primer for bonding of tri-n-butylborane resin to a leucite glassreinforced ceramic. J Prosthodont Res 57: 88-92.

9. Manicone PF, Rossi Iommetti P, Raffaelli L (2007) An overview of zirconia ceramics: basic and clinical properties to pplications. J Dent 35: 819-26.

10. R. Aguiar, CB Andre, CAG Arrais, AK Bedran-Russo, M. Giannini (2012) Micromorphology of resin-dentin interfaces using self-adhesive and conventional resin cements: A confocal laser microscope analysis. Inte J Adhes Adhesi 38: 69-74.

11. Zhang C, Degrange M (2010) Shear bond strengths of self adhesive luting resins fixing dentine to different restorative materials. J Biomater Sci Polym Ed 21: 593-608

12. Hervás Garcia A, Martinez Lozano Ma, Cabanes Vila J, Barjau Escribano A, Fos Galve P (2006) Composite reins. A review of the materials and clinical indications. Med Oral Patol Oral Cir Bucal 11: 215-20.

13. Garcia Fonsecal R, Dos Santos CA, Luis Adado G (2004) The influence of chemical activation on the hardness of dual-curing resing cements. Braz Oral Res 18 : $228-32$.

14. Poskus LT, Latempa AM, Chagas MA, Silva EM, Leal MP, Guimarães JG (2009) Influence of post-cure treatments on hardness and marginal adaptation of composite resin inlay restorations: an in vitro study. J Appl Oral Sci 17:617-22.

15. Acquaviva PA, Cerutti F, Adami G, Gagliani M, Ferrari M, Gherlone E, et al. (2009) Degree of conversion of three composite materials employed in the adhesive cementation of indirect restorations: a micro-Raman analysis. J Dent 37: 610-5.

16. Ritter AV, Ghaname E, Pimenta LA (2009) Dentin and enamel bond strengths of dual-cure composite luting agents used with dual-cure dental adhesives. J Dent 37:59-64.

17. Lee IB, An W, Chang J, Um CM (2008) Influence of ceramic thickness and curing mode on the polymerization shrinkage kinetics of dual-cured resin cements. Dent Mater 24: 1141-7.

18. Irie M, Maruo Y, Nishigawa G, Suzuki K, Watts DC (2010) Physical properties of dual-cured luting-agents correlated to early no interfacial-gap incidence with composite inlay restorations. Dent Mater 26: 608-15.

19. Annunziata M, Aversa R, Apicella A, Annunziata A, Apicella D, Buonaiuto C, et al. (2006) In vitro biological response to a light-cured composite when used for cementation of composite inlays. Dent Mater 22: 1081-5.

20. Arrais CA, Rueggeberg FA, Waller JL, de Goes MF, Giannini M (2008) Effect of curing mode on the polymerization characteristics of dual-cured resin cement systems. J Dent 36: 418-26.

21. Farrell CV, Johnson GH, Oswald MT, Tucker RD (2008) Effect of cement selection and finishing technique on marginal opening of cast gold inlays. J Prosthet Dent 99: 287-92.

22. Behr M, Rosentritt M, Wimmer J, Lang R, Kolbeck C, Bürgers R, et al. (2009) Self-adhesive resin cement versus zinc phosphate luting material: a prospective clinical trial begun 2003. Dent Mater 25: 601-4.

23. Karakaya S, Sengun A, Ozer F (2005) Evaluation of internal adaptation in ceramic and composite resin inlays by silicon replica technique. J Oral Rehabil. 32: 448-53.

24. Magne P, Cascione D (2006) Influence of post-etching cleaning and connecting porcelain on the microtensile bond strength of composite resin to feldspathic porcelain. J Prosthet Den 96: 354-61.

25. Ereifej N, Silikas N, Watts DC (2009) Edge strength of indirect restorative materials. J Dent 37: 799-806.

26. Yamamoto T, Nishiura R (2006) Marginal toughness of bonded dental ceramics evaluated by determination of the crack length. J Dent 34: 146-54.

27. Baroudi K, Silikas N, Watts DC (2008) Edge-strength of flowable resin-composites. J Dent 36: 63-8.

28. Kim SH, Watts DC (2007) In vitro study of edge-strength of provisional polymer-based crown and fixed partial denture materials. Dent Mater 23: 1570-3.

29. Nocchi Conceicao (2007) Conservative Dentistry. Health and Aesthetics, $2^{\text {nd }}$ edition, Brazil. Pan Am Med Pub.

30. Peumans M, Van Meerbeek BV, Lambrechts P, Vanherle G (2000) Porcelain venners: a review of the literature. J Dent 28: 163-77.

31. Soares CJ, Celiberto L, Dechichi P, Fonseca RB, Martins LR (2005) Marginal integrity and microleakage of direct and indirect composite inlays: SEM and stereomicroscopic evaluation. Braz Oral Res 19: 295-301.

32. Watts DC, Issa M, Ibrahim A, Wakiaga J, Al-Samadani K, Al-Azraqi M, et al. (2008) Edge strength of resin-composite margins. Dent Mater 24:129-33.

33. de Andrade OS, de Goes MF, Montes MA (2007) Marginal adaptation and microtensile bond strength of composite indirect restorations bonded to dentin treated with adhesive and low-viscosity composite. Dent Mater 23: 279-87.

34. Belli R, Pelka M, Petschelt A, Lohbauer U (2009) In vitro wear gap formation of self-adhesive resin cements: a CLSM evaluation. J Dent 37: $984-93$.

35. Ferreira MC, Vieira RS (2008) Marginal leakage in direct and indirect composite resin restorations in primary teeth: an in vitro study. J Dent 36: $322-5$.

36. Ilie N, Hickel R (2008) Correlation between ceramics translucency and thr ough Polymerization efficiency ceramics. Dent Mater 24: 908-14.

37. de Carvalho RF, Martins ME, de Queiroz JR, Leite FP, Ozcan M (2011) Influence of heat treatment on silane bond strength of resin cement to a feldspathic ceramic. Dent Mater 30: 392-7.

38. Hasegawa EA, Boyer DB, Chan DC (1991) Hardness of dual-cured cements under composite resin inlays. J Prothet Dent 66: 187-92.

39. Halhn P, Attin T, Grofke M, Hellwing E (2001) Influence od resin cement viscosity microleakage of ceramic inlays. Dent mater 17: 191-6. 
40. Jara Vidal P, Martinez Bello A, Correa Beltran G (2010) In vitro study of tensile strength of glass fiber posts cemented with four luting agents. Av Odontoestomatol 26: 255-62.

41. Mazzitelli C (2008) Evaluation of the binding of self-adhesive resin cements and dentin. Dent Material 24: $1156-63$.

42. Van Meerbeek B, Peumans M, Poitevin A, Mine A, Van Ende A, (2010) Relationship Between bond-strength tests and clinical outcom is. Dent Mater 26: 100-21.

43. Archegas LR, Freire A, Vieira S, Caldas DB, Souza MS (2011) Color stability and opacity of resin cements and flowable composites for ceramic veneer luting acce lerated after aging. J Dent 39: 804-10.

44. Atsu SS, MA Kilicarslan, Kucukesmen HC, Aka PS (2006) Effect of zirconium-oxide ceramic surface treatments on bond strength to adhesive resin. J Prosthet Dent 95: 430-6.

45. Yavuz T, Dilber E, Kara HB, Tuncdemir AR, AN Ozturk (2013) Effects of different surface treatments on shear bond strength in two different ceramic s ystems. Lasers Med Sci 28: 1233-9.

46. Gomes, Castillo-Oyagüe R, CD Lynch, J Montero, Albaladejo A (2013) Influence of sandblasting granulometry and resin cement composition on microtensile bond strength to zirconia ceramic for dental prosthetic frameworks. J Dent 2013: 310-6.

47. Braga R, Meira JB, Boaro LC, Xavier TA (2010) Adhesion to tooth structure: a critical review of "macro" test methods. Dent Mater 26: 38-49.

48. Armstrong S, S Geraldeli, Maia R, Raposo LH, Soares CJ, et al. (2010) Adhesion to tooth structure: a critical review of "micro" bond strength test methods. Dent Mater 26: 50-62.

49. Al-Assaf K, Chakmakchi M, Palaghias G, Karanika-Kouma A, Eliades G (2007) Interfacial characteristics of adhesive luting resins and composites with dentine. Dent Mater 23: 829-39.

50. Tsitrou EA, Helvatjoglu-Antoniades M, Van Noort R (2010) A preliminary evaluation of the structural integrity and fracture mode of minimally prepared resin bonded CAD/CAM crowns. J Dent 38: 16-22.

51. Minami H, Suzuki S, Murahara S, Saimi Y, Minesaki Y, Tanaka T (2009) Effect of fiber-premixed indirect resin composite substructure on fracture resistance of MOD composite inlays adhered with two different adhesive resin cements. Dent Mater J 28: 565-70.

52. Plotino G, Buono L, Grande NM, Lamorgese V, Somma F (2008) Fracture resistance of endodontically treated molars restored with extensive composite resin restorations. J Prosthet Dent 99: 225-32.

53. Jiang W, Bo H, Yongchun G, LongXing N (2010) Stress distribution in molars restored with inlays or onlays with or without endodontic treatment: a threedimensional finite element analysis. J Prosthet Dent 103: 6-12.

54. Magne P, Knezevic A (2009) Thickness of CAD-CAM composite resin overlays influences fatigue resistance of endodontically treated premolars. Dent Mater 25: $1264-8$.

55. Soares PV, Santos-Filho PC, Gomide HA, Araujo CA, Martins LR, et al. (2008) Influence of restorative technique on the biomechanical behavior of endodontically treated maxillary premolars. Part II: strain measurement and stress distribution. J Prosthet Dent 99: 114-22.

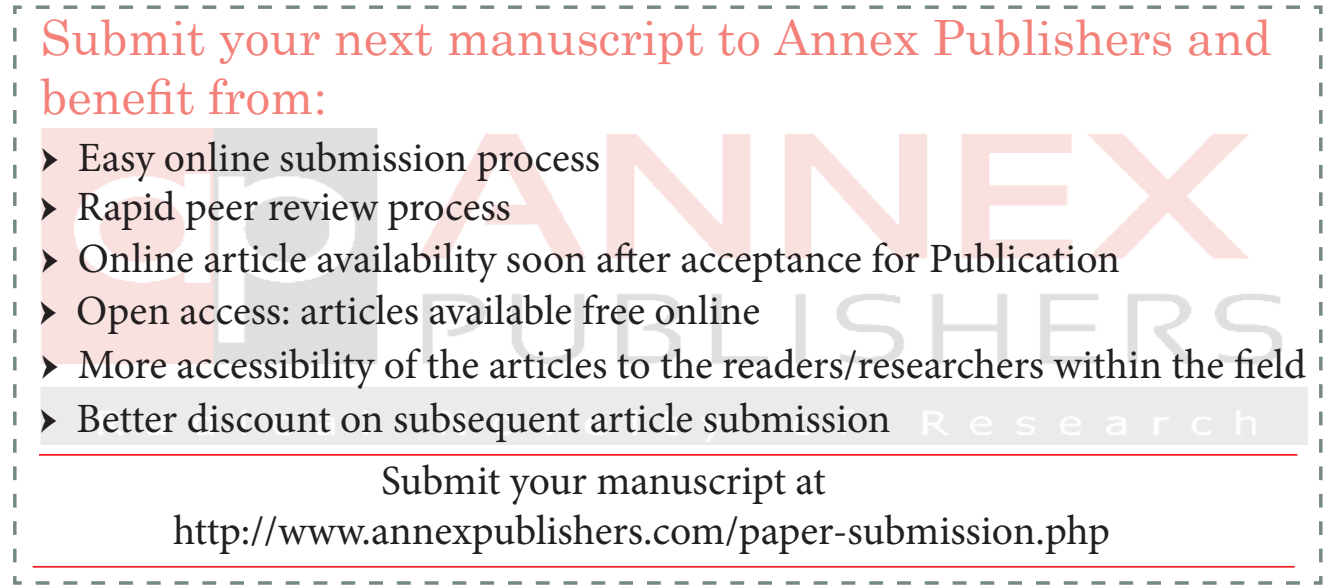

